\title{
Dynamics of Molecular Processes and the Sustainable Development of Humanity*
}

\author{
Yuan Tseh Lee ${ }^{1}$
}

\begin{abstract}
Yuan Tseh Lee was instrumental in the development and construction of an apparatus that utilized crossed molecular beams, presenting a break-through technique that allowed for the understanding of the dynamics of elementary chemical reactions. This was done by following the trajectories of reactants and reaction products in single collision events, allowing the visualization of the dynamics of how chemical reactions take place. This article also highlights Prof. Lee's belief in the severity of the consequences of global warming and his concerns relating to the need to substantially reduce carbon emissions.
\end{abstract}

Keywords: Dynamics; Molecular; Sustainable; Development; Humanity.

Thank you for inviting me to attend this exciting meeting. Earlier today you heard somebody mention that they liked water, well, I like baseball. My interest in baseball came about just after the Second World War, when I was in the third grade. At this time we were told that if you are good enough to make the baseball team that you would not have to attend the classes in the afternoon. This inspired me as I disliked going to class and as a result I became a very good second baseman. Consider the hitting of the baseball; this action resembles the scattering between two hard spheres. So the impact parameter, namely the aiming error, will determine whether you hit a home run, a pop-up or a ground ball. The aiming error is especially important as to the ball's trajectory. But if there is a force operating in between them, this will make things a little more complicated. For example if a molecule moves towards another molecule that has a repulsive force, it would be bounced backward. On the other hand, if the interaction is an attractive one then the molecule would bend over. So in one sense repulsion excludes the volume and attraction seems to expand the volume. And in the real world you are looking at the consequences of the interaction between atoms, or atoms and molecules. At a long distance, because of the dispersion force which is caused by the fluctuation of electrons density around the nuclei, the instantaneous dipoles created make the interaction between atom and molecule attractive. On the other hand, when the electron cloud moves close together it would become repulsive. So in the real world, if you look at the atom-molecule interaction, you will always find that the interaction is attractive at a long distance, and repulsive at a short distance. Then when you look at the scattering process between two atoms it becomes really interesting. If the impact parameter is zero it will bounce back, if you increase the aiming error it will become a line-drive and a home run and then gradually become a pop-up. But if you continue to increase the aiming error and observe the scattering angle, because of the attractive force becoming more important than the repulsive force, the scattering angle will move from positive to zero and then to negative, and after reaching a maximum value, it will move back to zero. This is what is happening in real atomic collisions. And that is really important. If you look at thermal-conductivity, viscosity of gaseous species and observe how they deviate from an ideal gas case, all these depend on the detail of scattering between atoms and molecules. These in fact depend on the interaction forces between atoms and molecules.

If you look at the chemical reaction, we know that the collision will bring about the chemical change. So if you mix hydrogen molecules and chlorine molecules, it will transform

\footnotetext{
${ }^{1}$ Institute of Atomic and Molecular Sciences, Academia Sinica, Taiwan. Published online 21 June 2017; doi:10.1142/S2529732517400065

*This article was transcribed from a presentation delivered by Professor Lee at the Molecular Frontiers Symposium in the Tokyo University of Science, Kagurazaka campus in 2016. It is published here in MFJ with permission from Professor Lee.

This is an Open Access article published by World Scientific Publishing Company. It is distributed under the terms of the Creative Commons Attribution 4.0 (CC-BY) License. Further distribution of this work is permitted, provided the original work is properly cited.
} 
into hydrogen chloride $\left(\mathrm{H}_{2}+\mathrm{CL}_{2} \rightarrow 2 \mathrm{HCL}\right)$. But as you know this equation only shows the stoichiometric relation between molecules, that of one molecule and one chlorine molecule being transformed into two hydrogen chlorine (HCL). However if you really mix hydrogen and chlorine together in the dark, although there are lots of collisions between $\mathrm{H}_{2}$ and $\mathrm{Cl}_{2}$, chemical change might not take place because it takes a very high collision energy to overcome the barrier to induce this change. On the other hand, if you shine light into the mixture, chlorine will dissociate and a new molecule, hydrogen chloride $(\mathrm{HCl}$ ) will be produced. Sometimes hydrogen chloride produced could be cold, sometimes it could be warm. If you follow the trajectories of the atoms and molecules, what you might see is the chlorine molecule dissociated into atoms and then the chlorine atom colliding with a hydrogen molecule to produce a hydrogen chloride and a hydrogen atom. This is the second step. The hydrogen atom produced will then react with a chlorine molecule and form $\mathrm{HCl}$ and $\mathrm{Cl}$. So $\mathrm{HCl}$ is produced in two ways, via the collision of the chlorine atom with hydrogen molecule, or the hydrogen atom with chlorine molecules as shown in Fig. 1.

So for most of us learning about chemical kinetics, we try to understand the mechanism and the specific rate constant of each step. These determine how a system moves systematically from reactant to product as a function of time. For humankind one of the most important chemical reactions is the combustion of hydrocarbon. This simple chemical reaction provides more than $80 \%$ of today's energy requirements. Of course, the oxygen-oxygen bond and carbon-hydrogen bond are weaker than the carbon-oxygen bond in $\mathrm{CO}_{2}$, and in the water molecule. That is why the transformation between methane and oxygen produce, carbon dioxide and water will produce a large amount of energy. As I said molecular collision induces chemical change, but again nothing will happen when you mix methane and oxygen unless you initiate the reaction. If you used a match to initiate the combustion process you will immediately see a bond rupture, addition of oxygen

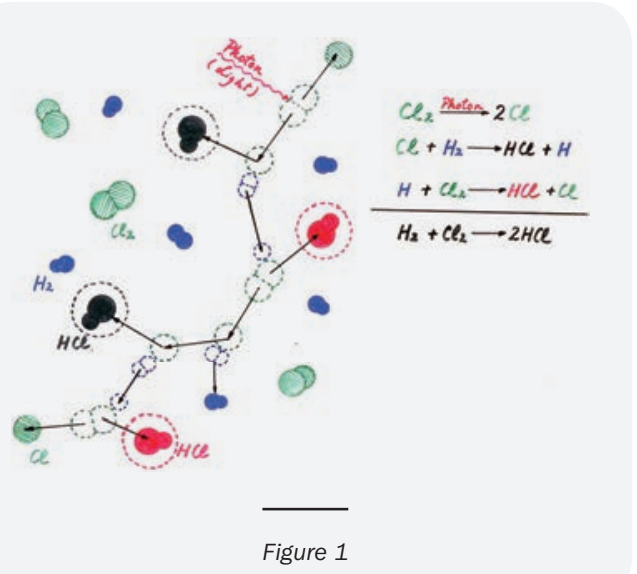

atom, dimerization, further elimination of hydrogen atoms, and step-by-step, carbon dioxide and the water molecule will be formed as end products. One of the final important steps is the reaction between carbon monoxide and hydroxyl radical $(\mathrm{OH})$ to produce $\mathrm{CO}_{2}+\mathrm{H}$.

The collision between carbon monoxide and a hydroxyl radical will form carbon dioxide and a hydrogen atom. This chemical reaction is quite interesting. The alignment of the molecule is important. However not only alignment is important, you also have to have enough energy to overcome the barrier. Otherwise, a chemical reaction will not take place as shown in Fig. 2. Over a hundred years ago, Arrhenius pointed out that the specific rate constant was dependent on temperature. In the Arrhenius equation $\left(K=A e^{-\varepsilon / k t}\right)$, the pre-exponential factor, or the A factor is actually determined by the probability of proper alignment and the activation energy $(\varepsilon)$ which determines that temperature dependence is related to the potential energy barrier. Arrhenius also pointed out over a century ago, that if man continued to burn fossil fuels that produce carbon dioxide, the surface temperature of the world would rise. But I don't think at that time he was worried about the consequences of global warming.

Now I want to take you through my work on molecular beam scattering experiments. As I pointed out earlier, if you look at the chemical reaction, $\mathrm{F}_{2}+\mathrm{D}_{2} \rightarrow 2 \mathrm{DF}$ as an example you are only observing the stoichiometric relation. The first thing that you need to understand is the mechanism. In this case, fluorine molecules represent a very weak bond and so it would dissociate into atoms quite easily. The fluorine atom would then react with deuterium molecules to form deuterium fluoride molecules, plus deuterium atoms. The deuterium atoms produced will then react with fluorine molecules to form deuterium fluoride and this chain reaction would complete the process, quite similar to the reaction between

\section{$\mathrm{CO}+\mathrm{OH} \rightarrow \mathrm{CO}_{2}+\mathrm{H}$}

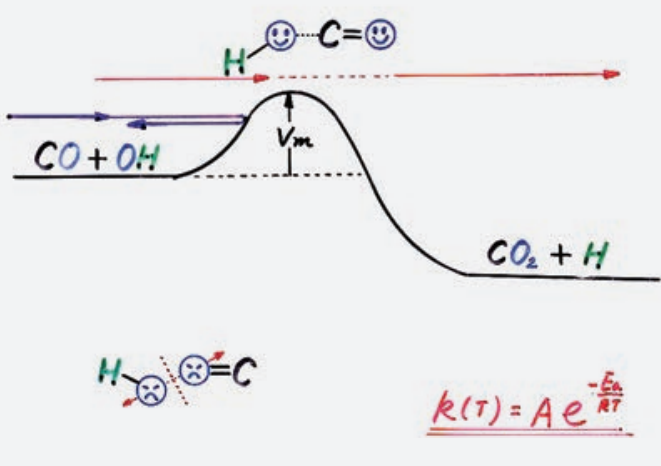

Figure 2 
hydrogen and chlorine. Make sure to be careful if you try this reaction yourselves, as it will explode. If the mechanism and specific rate are all known, then I can tell you the time-dependence of the chemical change, or how fast reactants will transform into products. The one thing that we really did not fully understand for a long time is how exactly elementary chemical reactions, as the consequence of single collision between atoms and molecules, take place.

However we did know that when a chemical reaction takes place, energy could be released. This release of chemical energy could be in the form of translational, rotational or vibrational. If our naked eye could see the movement of atoms and molecules, we would be able to understand the dynamics of elementary chemical reactions very easily. Unfortunately we cannot. However, in the laboratory it is possible to track the trajectories of atoms and molecules as shown in Fig. 3.

So about 50 years ago, when I was doing my postdoctoral research with Professor Herschbach at Harvard, scientists in Germany, England, The United States, all around the world, were already working on trying to observe the scattering between atoms and molecules and witness the chemical change in a single collision. Yet no one had managed to do this, except for the scattering of alkali atoms. I was young and when I was told that something was impossible, it became a challenge that got me really excited. In 1967, we started to design an apparatus to try to carry out this experiment. Among the important discoveries that I made during this time related to the adage that you need to learn from your mistakes and that this is how to make progress. But what I learned in addition is that it is not only essential to learn from your own mistakes but it is also important to learn from the mistakes of others. Just to realize that someone made a mistake is not helpful. You really have to gain knowledge through

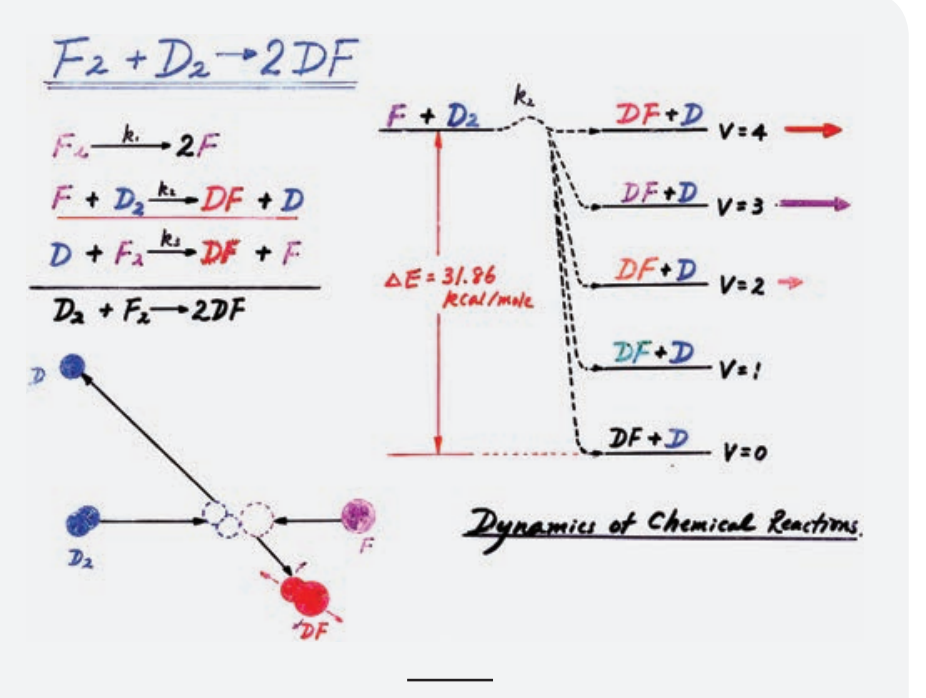

Figure 3 the mistakes that others have made. So I began to try to understand why all the eminent scientists around the world had not been able to do this experiment and solve the problems. And in doing this I began to realize that everyone who had attempted this had been misled. The discussion relating to the problems with the experiment had centered mostly in the crossing region of molecular beams in a vacuum chamber, where the number of molecules produced was very small. From there, only about 1 million molecules per second will reach the detector for electron bombardment Ionization detection. The ionization efficiency is only one in ten thousand. As a result, the focus at the time was on addressing the issue of increasing ionization efficiency. This meant that everybody who was working on the perceived problem in a crossed molecular beam experiment was trying to design a machine that would improve the detection efficiency. But no one had realized that in order to make the experiment successful, it was not only necessary to have an improved signal level, but also a good signal to noise ratio. When these 1 million molecules passed through the detector, it was possible to produce about 100 ions. This is a large enough number to be detected with an ion counter, if there is not several million times this in background ions to overwhelm the signal. Our machine was specially designed with a three-part pumping mechanism so the partial pressure of the interfering molecules would be reduced by about one million times, compared to that in the scattering chamber. The cut-out view of our machine is shown in Fig. 4. We adopted the configuration of fixed beams and rotatable detector for the measurements of angular and velocity distributions of scattered molecules. Our efforts represented the first time that the angular and velocity distributions of scattered molecules of any species could be measured. When we published the paper on this, the scientific community was astonished. Until then it had been believed that it was almost impossible to even observe the scattering of rare gas atoms. However we did succeed in building the machine that could do it. If one can measure differential scattering cross sections of argonargon scattering, one can derive the interaction potential, and from the interaction potential, you are able to calculate the macroscopic properties and transport phenomena of gaseous argon. As to the chemical reaction, the fluorine atom collided with the deuterium molecule and produced deuterium fluoride. When you scan the velocity and angular distributions that at low collision energy, as shown in Fig. 5, a fluorine atom and two deuterium atoms have to line up together, and the velocity distributions also indicate that most of the energy released is in the vibration of deuterium fluoride as the products move rather slowly. 


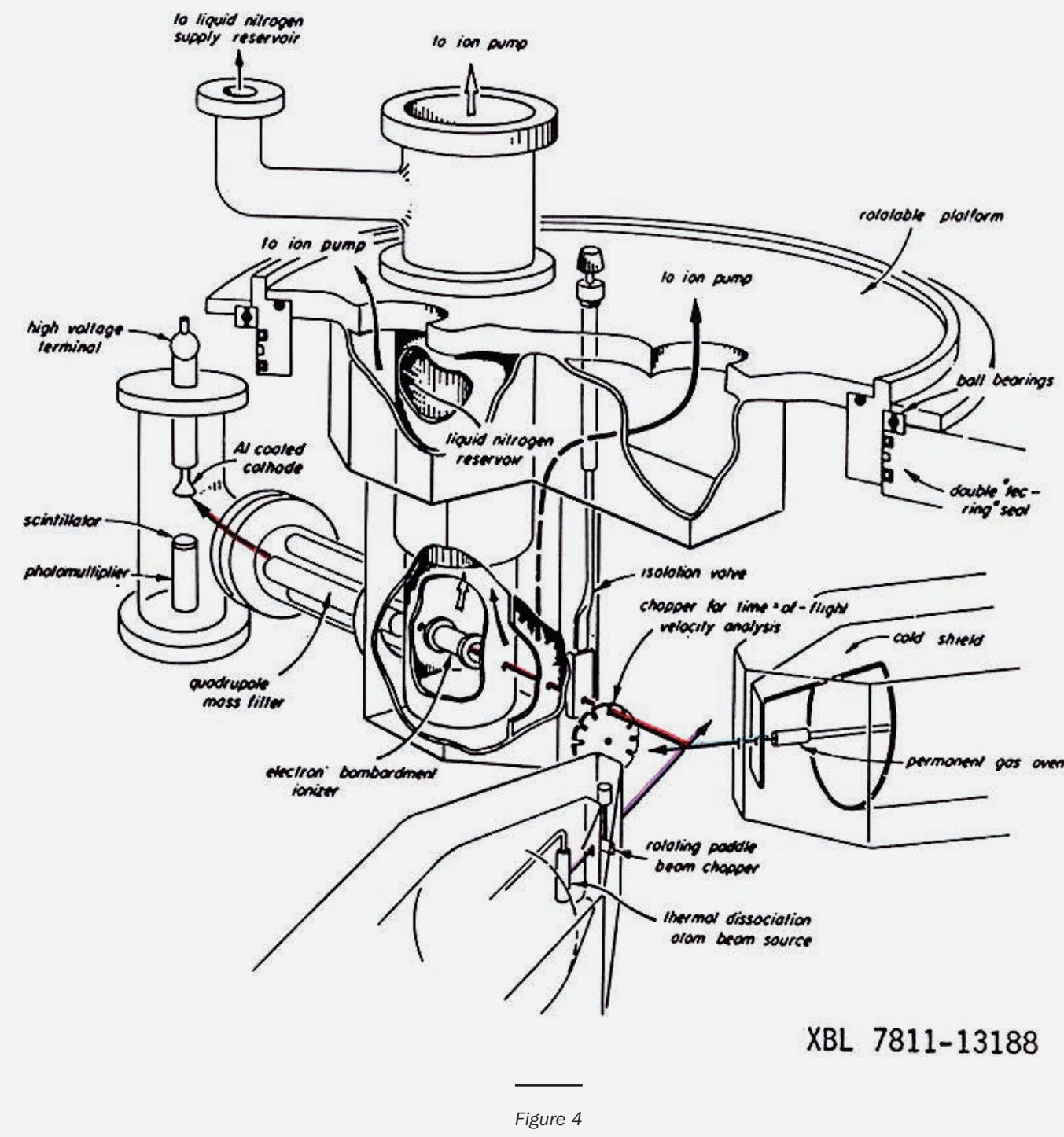

I would like to now address the question of the evolution of human society on earth. Our solar system was created about 9.5 billion years after the big bang. And about one billion years after the creation of the solar system, phenomena of life started on the earth's surface. After the formation of the solar system, it took about another 4.5 billion years to get us where we are today. Two million years ago, our ancestors appeared and evolved. One species, the homo sapiens survived and started to dominate and began to migrate all over the world. At the beginning the human population was small and did little to impact the global environment. It was not until the industrial revolution, when the steam engine, weaving machine and all the other machines were invented that things started to change drastically. I want to show you this painting from about 300 years ago of an aboriginal tribal wedding in Taiwan. Everything in the picture shown on the left hand side of Fig. 6 was derived from biomass. Everything was produced by sunshine. However, following the industrial revolution, what happened? Everything that we see today, concrete, steel, the fossil fuels used to produce these, are all dug out from the ground. Humankind used to be part of nature, however gradually we began to feel that we are the masters of nature. This dissociation and departure from nature is really causing a lot of problems. Earlier today the tremendous progress that took place in science and technology from 1850 until now was emphasized. The alarming thing is that alongside these reported technological advancements, we are burning fossil fuels in higher and higher quantities in tangent with the population explosion.

At the beginning of the last century we had 1.5 billion people, by its end we had 6 billion people. The population is currently estimated at around 7.2 billion. By 2050 the United Nations projects that 9.7 billion people will inhabit the planet. And with this growth in population and consumption, we have 


\section{$\mathrm{F}+\mathrm{D}_{2} \rightarrow \mathrm{DF}+\mathrm{D}, 1.82 \mathrm{kcal} / \mathrm{mole}$}

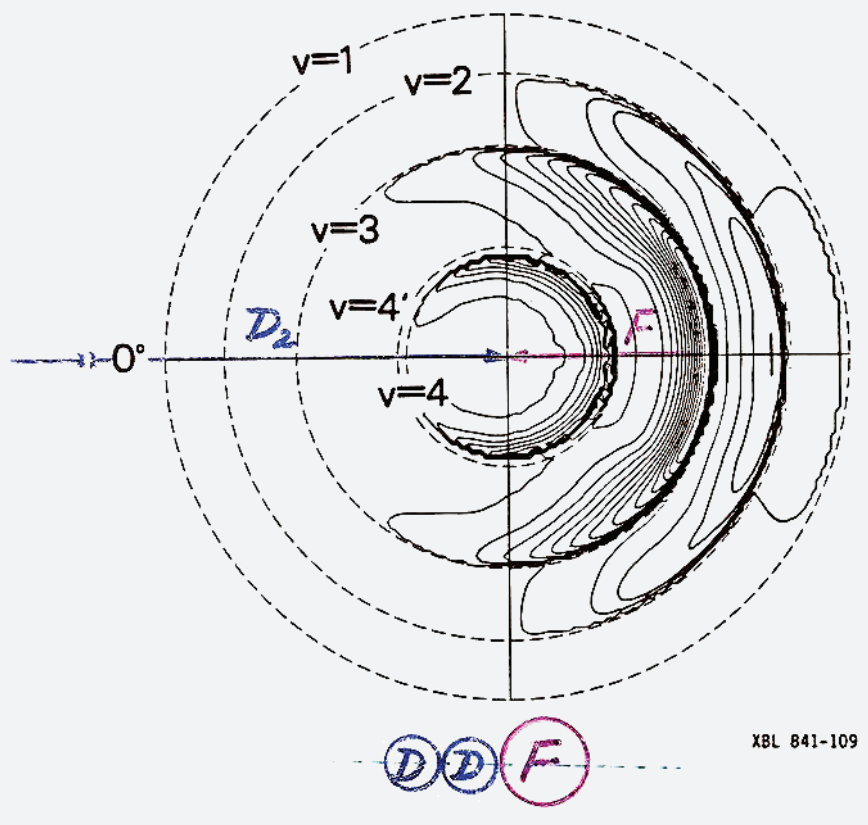

Figure 5

national borders without much hindrance, businesses are seeking out cheap labor sources for mass production. Countries like China, Vietnam and Indonesia provided cheap labor to produce consumer products for global consumption at the cost of using excessive natural resources. In the process we have destroyed the environment, created pollution and have caused $\mathrm{CO}_{2}$ emissions to keep on rising. Globalization and free trade have enabled that production and consumption proceed at full speed ahead, inspired by significant increases in GDP, heralded as progress. Whether you like it or not this is what we are doing at present. It's a horrible thing.

We are exceeding our planetary boundaries and are in a very precarious position. If you look carefully we are living in the very thin layers on the surface of the earth that hover above the earth. These are the hydrosphere and atmosphere. We have been living within these thin layers for thousands of years. The planet's natural processes allowed for a stable climate. However, we are now changing our living environment. The first thing you will notice is the pollution, especially if you visit

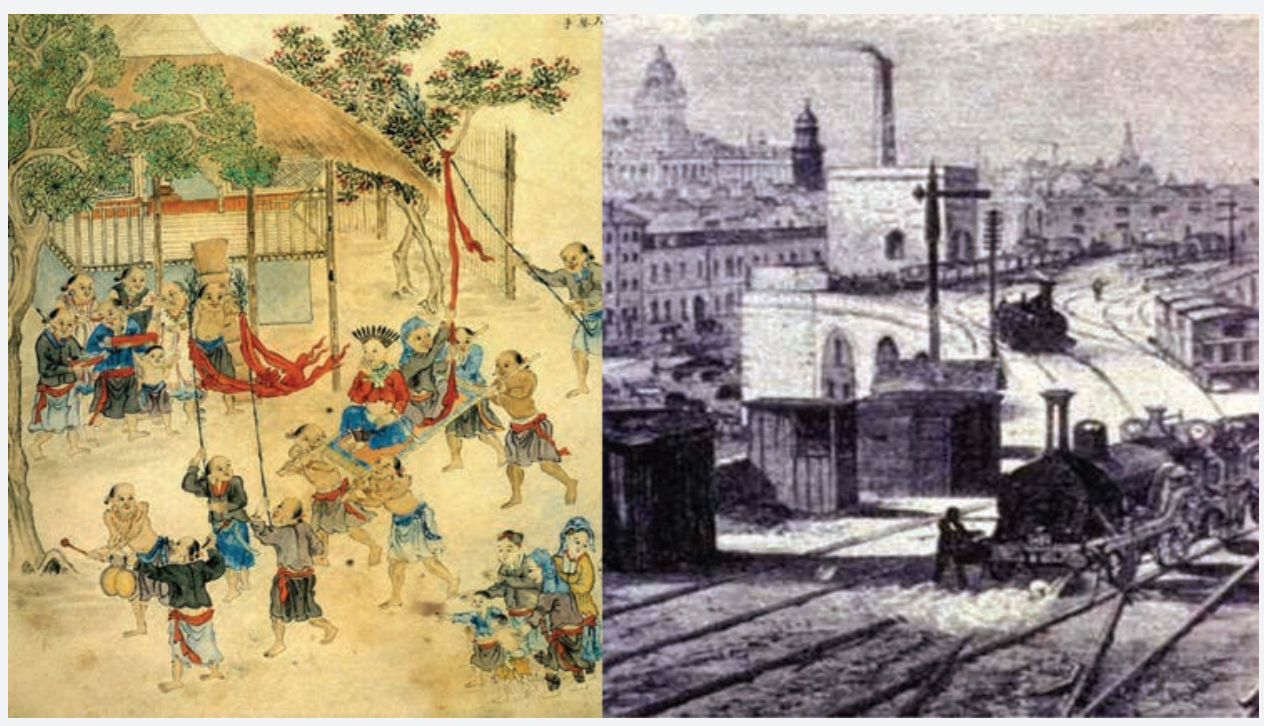

Figure 6

clearly overloaded the earth and with this we are changing the global environment. This is the reality of the civilization that we live in now.

You have heard about free trade and globalization. All over the world, with the capital and technology flow across many major cities in Asia. Can you convince yourself that breathing a heavily polluted air is progress? We are suffocating ourselves. I do not believe that no matter how rich we become that this really can be considered progress.

The second thing which is the most important, is the energy balance. Sunshine mostly as visible photons provides the planet of earth a large amount of energy and the earth also emits energy as infrared photons to the universe. If there were no greenhouse gases, i.e. carbon dioxide, methane, the temperature on the planet would be about minus 20 degrees. Prior to the industrial revolution, the planet's carbon dioxide 
concentration was maintained at about $280 \mathrm{ppm}$ for a long time, and at this level the global average temperature was stable at about 20 degrees. However now because greenhouse gas has increased we are trapping more energy than we are giving out, so that the temperature is rising, as seen in Fig. 7. Overall carbon dioxide concentration from the 1980s onwards has continued to go up. By the year 2000 this was up to $370 \mathrm{ppm}$ and it keeps going up and up. At present we are reaching concentration levels of 400 which are staggering and emissions are continuing to increase very fast. If you look at the period before the industrial revolution, the carbon dioxide concentration levels were maintaining at around $280 \mathrm{ppm}$. While there have been cyclical periods covering ice ages to warmer periods, these are due to the fact that the earth's orbit is not round and the axis of self-spinning makes an angle with the axis of the orbit. We are at the high point of the cyclic period and the surface temperature of the earth has never been so high. When the carbon dioxide concentration reaches a level of $450 \mathrm{ppm}$, the temperature will rise by 2 degrees centigrade from pre-industrial period which is considered to be the maximum temperature we should not go beyond. Otherwise, extreme weather events and abrupt climate change will threaten the existence of human civilization on earth.

In 2015 at the COP21 climate change conference in Paris, the parties that met agreed that humans have raised the temperature and that this is not good. Recently climate scientist James Hansen published a paper showing that this has gone up 1 degree centigrade already. It will be miserable if we allow the temperature to rise 1.5 degree above that of the pre-industrial era. So we are working with a very small margin. If you take a look at the IPCC report during the pre-industrial era, everything is fairly stable and extreme weather events are not so frequent. Compare this to now; the possibility of extreme weather events is already very high. What this actually means is that the situation is really quite serious already. Extreme weather events are becoming more common and increasingly severe. So while we keep on saying that the upper limit is a two degree centigrade increase. This is quite irresponsible for future generations because a twodegree centigrade rise would put the earth system in a very dangerous situation. However if we continue the business as usual, it is possible the rise in temperature will be as much as 4.5 degrees at the end of this century, and the humanity is not likely to survive under these conditions. Unless we start a deep decarbonization immediately, and become carbon neutral by the middle of the century. The temperature rise is most likely to exceed 1.5 degrees.

As I said we are already in a very undesirable situation. Consider the Yolanda typhoon, also called Haiyan typhoon which landed on the Philippines in 2013 with a wind speed of $100 \mathrm{~km} / \mathrm{h}$ or about $10 \mathrm{~m} / \mathrm{s}$, the most intense land falling tropical cyclone on record, killing 7000 people in one sweep. This type of weather related devastation is something which will happen with increasing frequency. When the leaders of 195 countries came to the COP21 meeting, they agreed that there was a need to swiftly decarbonize the energy system and negotiated a non-binding agreement for every country to work towards meeting a 2 degree centigrade upward limit. However it was said that ideally the limit should be set to a 1.5-degrees centigrade rise. Scientists will tell you that a 2-degree centigrade increase would be miserable. We really have to keep ourselves to this 1.5-degree centigrade as the upper limit. Other goals that were implemented included a pledge of one hundred billion dollars a year from developed countries towards helping developing countries, starting in 2020. This should be the relatively easy part of the agreement as global economic activity is around a hundred trillion dollars. This one 
hundred billion is a tiny sum of money. It was also agreed that in 2020 the carbon-reduction plans will be revisited.

What I'm saying here to you is that we are currently in a very dreadful predicament and even worse is that we do not have enough commitment to do things to save the next generation. I have served on the International Council of Science for many years as the President, this is an organization that is supposed to promote international science for the benefit of society. However, we are aware that in spite of the fact that civilization is heading towards a disaster, we are not doing enough. My advice to the younger generation is not to trust the older generation completely, when they tell you what to do, as they will not be there when the true crisis comes. The decision making should not be left to them.

Now I wish to summarize. Politically speaking, humanity is in the midst a global problem in need of global solutions. The United Nations however is nation-state based. There is no official mechanism to work together to implement guidelines for nations to do something really meaningful for humanity. Furthermore, from the social perspective, with too many people consuming too much and thereby overloading the earth, we are by definition over-developed. This over-developed world that has perilously taxed the planet does not have the right to dictate to the underdeveloped world the best way to proceed. The over-developed world needs to find different ways to reexamine so called development as well as finds means to turn down the impact of this. Today technologically the transformation from sunshine to electricity has become relatively inexpensive, but we have not yet learned how to store this electricity to make it available for the global market. So these are technological problems that we must learn to solve, transforming energy from the sun and storing this to make it available to the global market. Unfortunately, it seems that we are not progressing enough in this direction. We do not have a bright future ahead of us, and to change this we have to work hard, very hard. The first thing we have to do is to significantly decarbonize our society. By 2050, we must achieve carbon neutrality. While many scientists are doing everything they can, we need a total plan to realize this. Implementation of such a plan will not depend on science and technology alone. Society overall will have to transform. We must do this otherwise we will be very sorry. Well with this thought, I thank you very much for listening.

\section{Questions}

Audience: You mentioned that you measure angles and velocities to visualize molecular collisions. I wonder whether or not if it is possible to do same thing using super computers.

Yuan Tseh Lee: Yes certainly. Fifty years ago, when I worked on the helium scattering experiment, to determine the interaction potential, computers were not capable of such theoretical calculations and could not come up with a reliable result and could not even tell us whether helium dimer was in a bound state or not. But nowadays you don't even have to do the actual helium scattering experiment. You can just do the calculation to carry this out. This is true for even the reaction of a fluorine atom and hydrogen molecule. You can carry out the calculation and you will be able to tell what is going on. Certainly the present capabilities of supercomputing have enabled a very different way of finding the answers, and the interface between experiments and theoretical calculations have moved up to more complex systems.

Audience: I am interested in methane hydrate. However, if we access methane hydrate there may occur under-water disturbances that release methane into the air and promote global warming; however, methane hydrate has enormous possibilities and so shouldn't we get it and use methane hydrate?

Yuan Tseh Lee: Well, if we want to decarbonize human society, burning methane will not help. We should depend on sunshine. The sun provides enormous amounts of energy. In 1 hour, the amount of sun transmitted energy to the surface of the earth is about equivalent to the energy we use in 1 year. While methane hydrate produces lots of energy, we are better off not to burn it. People are concerned that when the ocean warms that this might cause methane hydrate to start to bubble out. In the year 2000 , a book was written that said that humanity will perish in 80 years and that part of the cause would be the increase of methane in the air as it released from the ocean and from the frozen tundra in Siberia. So it is not a good thing to use the methane hydrate. Instead we have to learn to store or transform the electricity generated by sunlight.

Audience: You said in your presentation that we, young people, have to research for next generation technologies for sustainable environments, but l've heard opinions that say that these types of technologies also pose threats to the environment. What do you think about such opinions?

Yuan Tseh Lee: This is why you often hear mention of both the light as well as the shadows of science and technology. While science and technology caused human society to develop we also found out that with so many people pursuing material comfort, that we are changing the environment. I have participated in many international programs on science and technology, giving people the promise that science and technology will make this place better. However, looking at the entire world situation today we might say that uncontrolled applications of science and technology have led towards the negative, knowing that we have a critical planetary boundary restriction to satisfy. In the application of science and technology one has to be careful. 
Audience: There are some scientists that assert that the global warming theory is not true and they claim that the recent global temperature rise is not due to human activity but rather due to the fact that the fluctuation of global temperature is cyclical and the present time is the period in the cycle when the temperature should be rising.

Yuan Tseh Lee: Well, if you trace temperature fluctuations backwards in time. You do see a glacial period, about every 20000 years, because the orbital motion of the earth around the sun is not circular and the spin of the earth makes an angle to the axis of orbital motion. For a certain period there will be an ice period. But the time for this is in thousands of years. Some people will say if you wait, the glacial cooling period will come. But how long can we afford to wait? We are going to wipe out humanity within a hundred years if we allow business as usual, so how can we wait for thousands of years. Scientists will tell you that the sea level rise is irreversible now and by the end of the century, there will be a multi-meter rise. Whether its $1 \mathrm{~m}$ or $2 \mathrm{~m}$ depends on how effective we are mitigating greenhouse emissions, but it can no longer be reversed. Scientists have studied every detail and although there are some more details that still need to be investigated, there is no longer a question as to the imbalance of energy coming in and going out and that the temperature of the surface of the earth is rising. This is why leaders of 195 Countries came together for COP21 because they realized it's important. The main problem is that circumstances and the ability to act continue to deteriorate.

Audience: What do you think about nuclear power as a way to fight depriving natural resources?

Yuan Tseh Lee: If you look at the current nuclear technology, it was built up more than 50 years ago and at that time and as it is today, people were worried about a proliferation of nuclear weapons. Current nuclear technology, from my point of view, is not a very ideal energy source. First of all, it is not safe enough. Secondly, we burn only about $2 \%$ of the uranium fuel and then $97-98 \%$ becomes waste, which is very difficult to manage. If every country were to work together, and come up with the next generation of nuclear power plant that would be safe and burn all the fuels and not produce radioactive waste, then you would not have the problem of weapons proliferation and this is not impossible.

Audience: Thank you very much. 